\title{
Sindrome de Stewart Treves: Relato de Caso
}

\author{
Stewart-Treves Syndrome: Case Report \\ Elisvania Rodrigues da Silva, Emmanuel Filizola Cavalcante \\ Francisco Valdeci de Almeida Ferreira, Lázaro Rogério Carvalho Soares \\ Sérgio Ferreira Juaçaba, Rogério Aguiar Ximenes
}

\begin{abstract}
RESUMO
Apresentamos um caso de linfoangiossarcoma de membro superior esquerdo, em paciente de 52 anos, previamente submetida à mastectomia radical modificada à esquerda, seguida de radioterapia devido ao carcinoma de mama 6 anos antes. Esta rara sindrome está associada a linfedema crônico, após mastectomia radical, seguida de radioterapia. Aproximadamente 400 casos foram relatados na literatura. A ocorrência infreqüente dessa doença e a aparência inócua do tumor, freqüentemente levam a retardo no diagnóstico $e$ tratamento. No caso dessa paciente, o diagnóstico foi obtido por meio de biópsia incisional da lesão, com confirmação pela imuno-histoquimica, utilizando marcadores de células endoteliais, anticorpo anti-CD31, vimentina e actina muscular. A paciente foi submetida à amputação do membro superior, e encontra-se em seguimento sem recorrência local ou à distância há 18 meses.
\end{abstract}

PALAVRAS CHAVES: Mama: câncer. Linfoangiossarcoma. Sarcoma. Radioterapia

\section{Introdução}

Os sarcomas vasculares ou angiossarcomas são neoplasias malignas que se originam de células com função, morfologia e características imunohistoquímicas de endotélio de vasos sanguíneo ou linfático, classificados com hemangiossarcoma e linfoangiossarcoma, respectivamente ${ }^{1}$.

Os angiossarcomas, associados ao linfedema crônico em extremidades, são morfologicamente similares aos outros angiossarcomas cutâneos ${ }^{2}$. Ocorrem em pacientes com linfedema adquirido, congênito ou traumático, mais comumente após mastectomia que inclua dissecção dos linfonodos axilares, seguida de radioterapia, para tratamento do câncer de mama ${ }^{3}$. Na presença de linfedema, os angiossarcomas crescem como placas coalescentes ou nódulos cutâneos e subcutâneos. Embora a natureza vascular específica dessa lesão seja questionada, estudos imuno-histoquímicos

Hospital do Câncer do Ceará - Instituto do Câncer do Ceará Correspondência:

Elisvania Rodrigues da Silva

Rua Professor Heráclito, $\mathrm{n}^{\circ} 440$ apto. 401 - Papicu

60175-595 - Fortaleza - CE

e-mail: lazrog@ig.com.br confirmam a origem endotelial ${ }^{4}$. Em 1960, a incidência de linfoangiossarcoma entre as pacientes submetidas à mastectomia radical, variava de 0,07 a $0,45 \%{ }^{5}$, após cinco anos de seguimento. Atualmente, devido à mudança na técnica operatória para o câncer de mama, houve diminuição do linfedema crônico e, portanto, menor incidência dessa sindrome ${ }^{5}$. A literatura registra, aproximadamente, 400 casos de angiossarcoma associado a linfedema crônico, dos quais 360 ocorreram após mastectomia ipsilateral ${ }^{6}$.

\section{Relato de caso}

Paciente 52 anos de idade, havia sido submetida, em 1996, à mastectomia radical modificada à esquerda, por carcinoma ductal infiltrante da mama. A cirurgia foi seguida de radioterapia adjuvante da parede torácica (plastrão) e fossa supraclavicular ipsilateral. Em março de 2001, a paciente retornou ao ambulatório de Mastologia do Hospital do Câncer-Instituto do Câncer do Ceará, referindo edema do membro superior esquerdo, presente há cinco anos. Notou aumento progressivo do volume do braço, acompanhado de le- 
são vinhosa, surgida há cinco meses (Figura 1). $\mathrm{Na}$ oportunidade, negava dor local ou prurido. Ao exame físico, constatou-se linfedema crônico, acompanhado de lesão vinhosa de aspecto erisipelióide endurecida, no terço superior do membro superior esquerdo medindo, 13 por $7 \mathrm{~cm}$ (Figura 2).

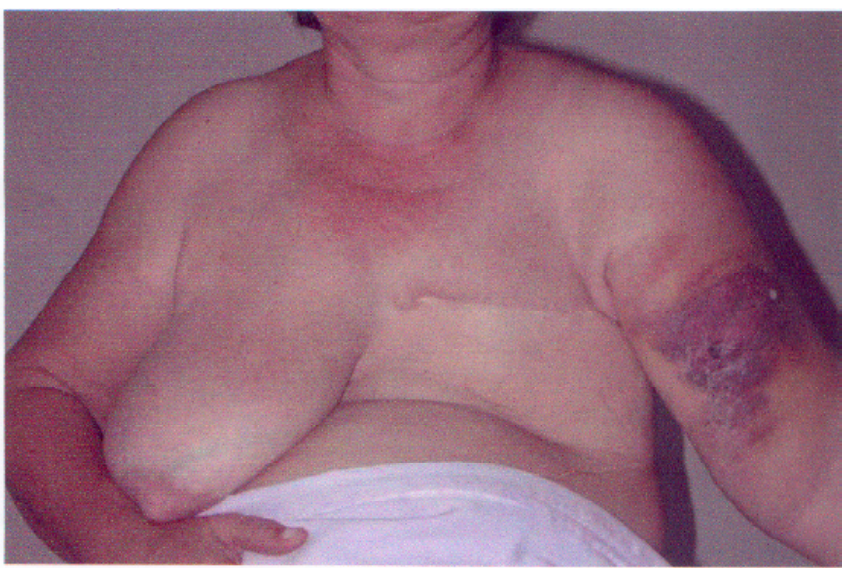

Figura 1-Notar mastectomia e linfedema no membro superior esquerdo ipsilateral, associado à lesão vinhosa no terço superior.

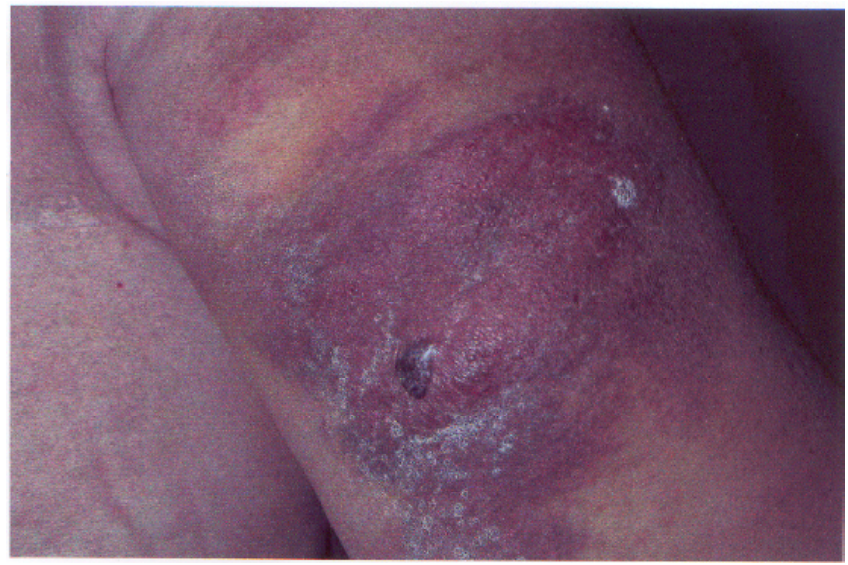

Figura 2 - Notar o aspecto erisipelióide da lesão de coloração vinhosa, associado ao linfedema crônico do membro superior esquerdo.

Diante do quadro, foi indicada biópsia incisional da lesão, sob anestesia geral. O exame anatomopatológico evidenciou, à microscopia: neoplasia heterotipica, formada por canais vasculares sanguíneos pletóricos e aglomerados nodulares de células endoteliais atípicas, em torno de fendas. Foi então, realizado estudo imunohistoquímico, utilizando marcadores de células endoteliais, vimentina, actina muscular e anticorpos anti-CD45 e CD31. O resultado do estudo revelou tratar-se de linfoangiossarcoma, considerando que as células tumorais foram marcadas como endoteliais (CD31) situadas em fendas ou vasos, enquanto a parede vascular foi marcada com a actina muscular (Figura 3). Por oportuno, solicitou-se tomografia do tórax e ultra-sonografia abdominal, nenhum dos exames, evidenciando, doença metastática.

A paciente submeteu-se à amputação do membro superior esquerdo, sob anestesia geral. O exame anatamopatológico foi compativel com linfoangiossarcoma associado a linfedema crônico, sem infiltração do plano ósseo ou medula óssea. A paciente vem sendo acompanhada ambulatorialmente, há cerca de um ano e seis meses, sem recidiva local ou à distância, por meio de exame clínico e radiológico (radiografia de tórax).

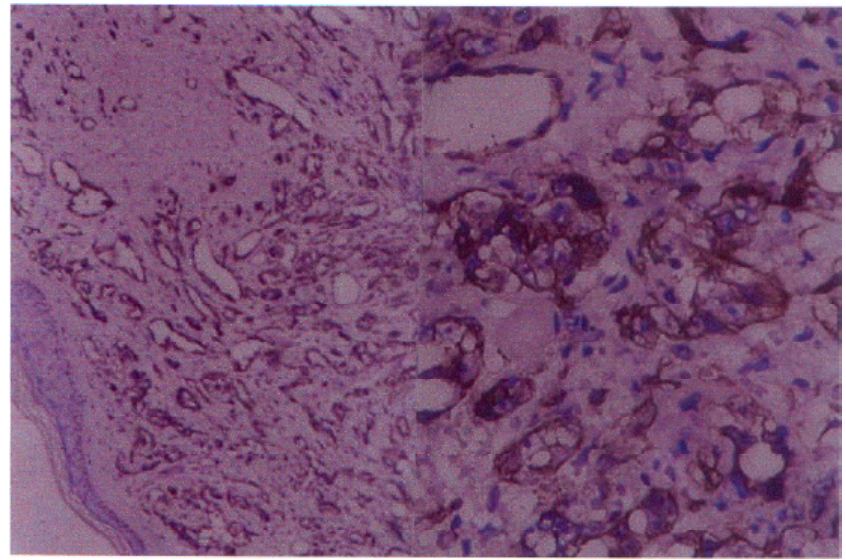

Figura 3 - Notar espaços vasculares dilatados, irregulares e de tamanhos variados revestidos por células poligonais com núcleos hipercorados. Células endoteliais marcadas com anticorpos anti-CD31 no campo de 100x e 200x.

\section{Discussão}

Tradicionalmente, os linfoangiossarcomas são definidos como sarcomas vasculares, associados ao linfedema crônico. É geralmente aceito que os linfoangiossarcomas não estão relacionados, diretamente, ao carcinoma mamário, mas que se desenvolvem, espontaneamente, como neoplasia primária, na área do linfedema crôni$\mathrm{co}^{7}$. Vários tipos de linfedema crônico, tais como congênito, idiopático, adquirido e traumático, podem estar associados a este tumor maligno ${ }^{1-8}$. Os linfoangiossarcomas ou sarcomas vasculares são neoplasias malignas que se originam de células com função, morfologia e características imunohistoquímicas de endotélio. Áreas previamente submetidas à radiação ionizante apresentam tendência maior ao desenvolvimento de sarcomas, a longo $\mathrm{prazo}^{8,9}$. O tempo de latência, entre o tratamento para câncer de mama e o diagnóstico da síndrome de Stewart-Treves é de 10 a $20 \operatorname{anos}^{5}$. A maior incidência dessa sindrome ocorre na faixa etária compreendida entre a quinta e a sétima 
década de vida, quando também é maior a incidência de câncer de mama ${ }^{10}$.

Cozen et al. ${ }^{9}$ conduziram estudo do tipo casocontrole avaliando a relação entre o câncer de mama invasivo e o angiossarcoma de membro superior, encontrando odds ratio de 59,3, mostrando que há risco aumentado para o surgimento dessa neoplasia vascular em pacientes submetidas à mastectomia, devido ao câncer de mama, quando estabelecida comparação com mulheres que não tinham o diagnóstico de câncer de mama. O linfedema pós-mastectomia por câncer de mama, conseqüente ao bloqueio linfático e/ ou radioterapia, tem sido postulado como fator de risco para o desenvolvimento de sarcomas de partes moles ${ }^{9}$. Os autores relatam que o tipo histológico encontrado com maior freqüência, entre os sarcoma de partes moles, foi o linfoangiossarcoma de membro superior $(70 \%)$, e que o intervalo médio entre o diagnóstico de câncer de mama e o angiossarcoma de membro superior foi de 9,7 anos ${ }^{9}$.

A falta de maior conhecimento sobre essa neoplasia, aliada à sua aparência inócua, leva ao retardo do diagnóstico. Clinicamente, são lesões avermelhadas, azuis ou púrpuras, moles e esponjosas e, microscopicamente, os espaços vasculares são irregulares, de tamanhos variados e revestidos por células poligonais, com núcleos hipercorados, com pleomorfismo nuclear ${ }^{4-8}$. Os angiossarcomas são neoplasias pouco diferenciadas e, freqüentemente, requerem confirmação pela imuno-histoquímica. Demonstram positividade imuno-histoquímica para marcadores de células endoteliais, tais como fator VIII, anticorpos antiCD34 e CD31, sendo que o último é o que mais apresenta sensibilidade e especificidade ${ }^{1,2}$. O angiossarcoma é um tumor de alto grau de malignidade (G3), tanto em sua localização visceral, como em partes moles, sendo, portanto, o tumor vascular maligno de pior prognóstico ${ }^{1}$.

A sintomatologia mais comum dos tumores vasculares localizados nas partes moles é o aparecimento de nódulo, com ou sem dor, não sendo raro ocorrer diagnóstico errôneo de hematoma ou abscesso devido à raridade desse tipo de lesão ${ }^{1}$. A avaliação pulmonar é fundamental, pelo fato de o pulmão ser o órgão preferencial de ocorrência das metástases. O diagnóstico histopatológico deve ser dado por biópsia ampla, a céu aberto, sempre que possível com anestesia geral, bloqueio medular ou de plexo nervoso, evitando as infiltrações para anestesia local $^{1}$. O diagnóstico diferencial deve incluir outras neoplasias vasculares e epiteliais, sarcomas e melanoma maligno. O tratamento inclui cirurgia, radioterapia e quimioterapia. No angiossarcoma de origem endotelial, o tratamento no entanto, restringe-se praticamente à cirur- gia, visto que não há resposta à radio ou à quimioterapia. As cirurgias indicadas para os pacientes com tumores vasculares malignos variam de acordo com a possibilidade de preservação do membro e, não sendo possivel a sua conservação, o procedimento indicado é a amputação ${ }^{1}$. A excisão ampla deve ser feita, como em outros sarcomas de extremidades, com $2-3 \mathrm{~cm}$ de margem de tecido normal, isso quando possível. O envolvimento ósseo, nervoso ou vascular é indicação relativa para proceder à amputação ${ }^{11}$.

A radioterapia tem sido usada, freqüentemente, como tratamento adjuvante, curativo ou paliativo. Kaufmann et al. ${ }^{12}$ recomendam, no seu estudo, que em lesões pequenas e limitadas, sejam usadas altas doses de irradiação, variando de 5500 a 6000 cGy, divididas em 27-30 frações, por periodo de seis semanas. Os autores relatam também a sua experiência com a utilização de quimioterapia (actinomicina D $2 \mathrm{mg}$ intravenosa), associada à radioterapia, após tratamento cirúrgico, apresentando bons resultados, após dois anos de seguimento. Atualmente, o agente quimioterápico mais freqüentemente utilizado para tratamento de sarcomas vasculares, é a doxorrubicina isolada ou associada a outros agentes ${ }^{13}$. Grobmyer et al. ${ }^{13}$, no seu trabalho de revisão da literatura, avaliando 92 casos dessa síndrome, concluíram que excisão local ampla é equivalente à amputação do membro e que a quimioterapia, com ou sem radioterapia, tem sido reservada para tratamento de pacientes com doença inoperável, que desenvolveram recorrência, ou que tenham recusado a amputação.

Referidos tumores vasculares malignos têm, como característica capilares com células neoplásicas, causando metástases preferencialmente hematogênicas, para os pulmões, da mesma forma como acontece com os sarcomas, em geral ${ }^{1}$. Chung et al. ${ }^{7}$ relatam dois casos de linfoangiossarcoma de extremidade pós-mastectomia, em que uma das pacientes apresentava metástase pulmonar, detectada pela tomografia computadorizada do tórax, no momento do diagnóstico. Esta veio a falecer sete meses após a conclusão de três ciclos de quimioterapia paliativa. Os autores observaram sobrevida, após o diagnóstico dessa neoplasia vascular, variando de 8 a 15 meses. Grobmyer et al. ${ }^{13}$ encontraram sobrevida média, para pacientes com essa doença, de 20 meses. Por sua vez, Aygit et al. ${ }^{14}$ relatam um caso dessa sindrome, que veio a óbito seis meses após a amputação do membro superior, pondo em evidência que o tratamento dessa síndrome é tido como insatisfatório. O seguimento dessas pacientes deve ser feito por meio de rastreamento de metástase, por tomografia computadorizada de tó- 
rax e, se isso não for possivel, por uma radiografia de tórax ${ }^{1}$.

A paciente, no caso deste estudo, encontrase em seguimento há um ano e seis meses, sem recidiva local ou à distância. Em linhas gerais, para um planejamento terapêutico adequado, é fundamental analisar as condições clínicas de cada paciente, o estadiamento da doença e conhecimento da história natural da neoplasia, indicando, de forma precisa, os exames complementares e, finalmente, a biópsia, com exame histopatológico. Conclui-se que, apesar da raridade dessa síndrome e de seu mau prognóstico, o diagnóstico precoce, associado à cirurgia radical, pode possibilitar melhor sobrevida.

\section{ABSTRACT}

The authors present a case of lymphangiosarcoma in the upper left limb of a 52-year-old patient previously submitted to a left-sided modified radical mastectomy followed by radiotherapy due to breast cancer six years earlier. This rare syndrome is associated with chronic lymphedema as a consequence of radical mastectomy followed by radiotherapy. Approximately 400 cases have been reported in the literature. The infrequent occurrence of this disease and the rather innocuous appearance of the tumor often lead to late diagnosis and treatment. In the present case, the diagnosis was based on an incision biopsy of the lesion and confirmed immunohistochemically using endothelial markers, antibodies (anti-CD31), vimentin and muscle actin. The patient's limb was amputated and no local or distant recurrence has so far been observed during 18 months of follow-up.

KEYWORD: Breast: neoplasms. Lymphangiosarcoma. Sarcoma. Radiotherapy.

\section{Referências}

1. De Vita VT, Hellman S, Rosenberg AS. Sarcomas of the soft tissues and bone. In: De Vita VT, Hellman S, Rosenberg AS, editors. Cancer: principles and practice of oncology. $6^{\text {th }}$ ed. Philadelphia: Lippincott Williams \& Wilkins; 2001. p.1841-83.
2. Damjanov I, Linder J. Soft - tissue tumors. In: Damjanov I, Linder J, editors. Anderson's Pathology. 10 ${ }^{\text {th }}$ ed. St Louis: Mosby; 1996.p.2500.

3. Rosai J. Soft tissues. In: Ackerman's surgical pathology. $8^{\text {th }}$ ed. St Louis: Mosby; 1989. p.2070.

4. Sternberg SS, Antonioli DA, Carter D, Mills SE, Oberman HA. The Breast. In: Sternberg SS, Antonioli DA, Carter D, Mills SE, Oberman HA, editors. Diagnostic Surgical Pathology. $3^{\text {rd }}$ ed. Philadelphia: Lippincott Williams \& Wilkins; 1999. p.368-9.

5. Heitman C, Ingianni G. Stewart-Treves syndrome: lymphangiosarcoma following mastectomy. Ann Plast Surg 2000; 44:72-5.

6. Bisceglia M, Attino V, D’Addetta C, Murgo R, Fletcher CD. La sindrome de Stewart-Treves in fase precoce: presentazione di due casi e revisione della letteratura. Pathologica 1996; 88:483-90.

7. Chung KC, Kim HJ, Jeffers LL. Lymphangiosarcoma (Stewart-Treves syndrome) in postmastectomy patients. J Hand Surg [Am] 2000; 25:1163-8.

8. Fletcher CDM. Tumors of blood vassels and lymphatics. In: Fletcher CDM, editor. Diagnostic Histopathology of Tumors. $1^{\text {st }}$ ed. New York: Churchill Livingstone; 1995. p.64-5.

9. Cozen W, Bernstein L, Wang F, Press MF, Mack TM. The risk of angiosarcoma following primary breast cancer. Br J Cancer 1999; 81:532-6.

10.Devolder S, Breuillard F, Gross S. Guess What! Lymphangiosarcoma of Stewart-Treves. Eur J Dermatol 1998; 8:527-8.

11.Lewis JJ, Brennan MF. Soft tissue sarcomas. Curr Probl Surg 1996; 33:817-72.

12.Kaufmann T, Chu F, Kaufmann R. Postmastectomy lymphangiosarcoma (Stewart-Treves syndrome): report of two long-term survivals. Br J Radiol 1991; 64:857-60.

13.Grobmyer SR, Daly JM, Glotzbach RE, Grobmyer AJ $3^{\text {rd }}$. Role of surgery in the management of postmastectomy extremity angiosarcoma (Stewart-Treves syndrome). J Surg Oncol 2000; 73:182-8.

14.Aygit AC, Yildirim AM, Dervisoglu S. Lymphangiosarcoma in chronic lymphoedema. Stewart-Treves syndrome. J Hand Surg [Br] 1999; 24:135-7. 